\title{
Epigallocatechin gallate inhibits human tongue carcinoma cells via HK2-mediated glycolysis
}

\author{
FENG GAO ${ }^{1,2,5}$, MING LI ${ }^{3}$, WEN-BIN LIU ${ }^{4}$, ZHONG-SU ZHOU ${ }^{3}$, \\ RUI ZHANG ${ }^{3}$, JUN-LING $\mathrm{LI}^{2}$ and KE-CHAO ZHOU ${ }^{1}$ \\ ${ }^{1}$ Powder Metallurgy Research Institute of Central South University; ${ }^{2}$ Department of Ultrasonography, \\ The Third Xiangya Hospital of Central South University; ${ }^{3}$ Changsha Stomatological Hospital; \\ ${ }^{4}$ Department of Pathology, The Affiliated Cancer Hospital of Xiangya School of Medicine, \\ Central South University, Hunan Cancer Hospital; ${ }^{5}$ Postdoctoral Station of Materials Science \\ and Engineering of Central South University, Changsha, Hunan, P.R. China
}

Received September 2, 2014; Accepted December 4, 2014

DOI: $10.3892 /$ or.2015.3727

\begin{abstract}
Epigallocatechin gallate (EGCG), one of the major catechins found in green tea, was suggested to play a role as a chemopreventive agent in various human cancer models. In this study, we reported that EGCG has a profound antitumor effect on human tongue carcinoma cells by directly regulating glycolysis. EGCG dose-dependently inhibited anchorage-independent growth and short-term EGCG exposure substantially decreased EGF-induced EGF receptor (EGFR), Akt and ERK1/2 activation, as well as the downregulation of hexokinase 2 (HK2). Furthermore, inhibition of EGCG-mediated HK2 expression was involved in Akt, but not in ERK1/2 signaling pathway suppression. Overexpression of constitutively activated Akt1 rescued inhibition of EGCG-induced glycolysis. Moreover, EGCG inhibited HK2 expression on mitochondrial outer membrane and induced apoptosis. In summary, the results suggested that EGCG or a related analogue, may have a role in the management of human tongue carcinoma.
\end{abstract}

\section{Introduction}

Epidemiologic studies indicate that green tea consumption decreases cancer risk (1-3). Different tea preparations contain varying amounts of polyphenols and epigallocatechin gallate (EGCG) is the most abundant, best-studied and possibly most

Correspondence to: Dr Ming Li, Department of VIP Center, Changsha Stomatological Hospital, Youyi Road 389, Tianxin, Changsha, Hunan 410004, P.R. China

E-mail: minglibio@163.com

Abbreviations: EGCG, epigallocatechin gallate; EGFR, EGF receptor; VDAC, voltage-dependent anion channel; PARP, poly(ADPribose) polymerase; HK2, hexokinase 2

Key words: tongue carcinoma, EGCG, HK2, Akt potent polyphenol against cancer found in green tea (4-6). EGCG reportedly exerts cancer preventive activity at a variety of organ sites, including oral cavity, esophagus, stomach, colon, skin, lung, pancreas and mammary gland (5,7-10). Previous studies suggested that EGCG affects different signal transduction pathways, including inhibition of various protein kinases $(11,12)$, suppression of the activation of transcription factors such as AP-1 $(13,14)$ and NF- $\mathrm{B}(15,16)$, blockade of growth factor receptor-mediated pathways, and induction of cell-cycle arrest or apoptosis $(16,17)$. EGCG was also found to inhibit cell transformation (18), and repress angiogenesis in various cancer models $(8,19,20)$. However, the mechanisms explaining the cancer preventive activity of EGCG remain to be elucidated.

Carcinoma of the oral tongue is the most common cancer in the oral cavity (21). The cause and incidence of tongue carcinoma are associated with different factors, such as genetic (mutations in DNA oncogenes and/or tumor-suppressor genes) and environmental (chemical, physical, smoke and alcohol) (21-23). Oral tongue carcinoma has a high propensity of lymph-node metastasis, even in early local diseases. The incidence of occult nodal metastasis is reportedly 30-40\% (24-26). Treatment of tongue carcinoma and conservation of important functions such as language articulation, swallowing and respiration remain a challenge. The overall 5-year disease-free survival rate of tongue carcinoma has not changed substantially over the past decades and has been estimated to be $\sim 50 \%(26,27)$. A major challenge in treating tongue carcinoma is to identify novel therapeutic targets or develop new anticancer agents that can complement current surgical treatment.

In general, even in the presence of oxygen, most tumor cells are predisposed to consume glucose through the glycolytic pathway as their major energy source to rapidly generate ATP and biosynthetic intermediates to sustain unlimited growth (28). This phenomenon, which is defined as the Warburg effect, has been consistently observed in most types of cancer, and is considered to be an emerging hallmark of cancer (29). The canonical oncogenic signaling pathways and transcription factors, including PI3-K/Akt, c-Myc and hypoxia-inducible factor 1 (HIF1), are involved in 
the regulation of numerous genes that are responsible for the metabolic difference in the solid tumor, such as hexokinase 2 (HK2), lactate dehydrogenase A and cell transporters (30-32). $\mathrm{HK} 2$, a rate-limiting enzyme of glycolysis, is upregulated in a number of cancers. HK2 overexpression is associated with greater glucose consumption and increased macromolecular biosynthesis. HK2 now has been recognized as a therapeutic target in various types of cancer (33-35).

In the present study, we first reported that HK2 is a key modulator of EGCG-induced glycolysis suppression in human tongue carcinoma cells. We evaluated its underlying mechanism of action and demonstrated that the Akt signaling pathway is involved. The findings suggest that targeting metabolic enzymes may be a novel preventive and therapeutic target in this type of tumor.

\section{Materials and methods}

Cell culture and transfection. The Tca8113 and TSCCa human tongue squamous cell carcinoma cell lines were purchased from the China Center for Type Culture Collection (Wuhan University) and cultured with Dulbecco's modified Eagle's medium (DMEM) containing 10\% fetal bovine serum (FBS) and 1\% antibiotics. For transfection experiments, the Lipofectamine ${ }^{\mathrm{TM}} 2000$ transfection reagent (Invitrogen, Carlsbad, CA, USA) was used according to the manufacturer's instructions.

Reagents and antibodies. EGCG was obtained from Sigma (St. Louis, MO, USA), and Myr-Akt1 was purchased from Addgene (Cambridge, MA, USA). Wortmannin, PD98059, anti-p-EGFR (Tyr1068), anti-Akt (pan), anti-Akt1, anti-p-Akt (S473), anti-ERK1/2, anti-p-ERK1/2 (Thr202/Tyr204), antiHK2, anti-cleaved-PARP, anti-caspase 3 and anti- $\alpha$-tubulin antibodies were purchased from Cell Signaling Technology, Inc. (Danvers, MA, USA). Anti- $\beta$-actin, anti-rabbit IgG-HRP and anti-mouse IgG-HRP were purchased from Santa Cruz Biotechnology, Inc. (Santa Cruz, CA, USA). Anti-VDAC was purchased from Abcam (Cambridge, UK).

Measurement of glucose uptake and lactate production. Cells $\left(5 \times 10^{5}\right)$ were seeded in 6-well plates. After incubation for $4 \mathrm{~h}$, the culture medium was discarded and the cells were incubated in fresh medium for another $8 \mathrm{~h}$. Glucose and lactate levels were measured using the Automatic biochemical analyzer (AU680; Beckman Coulter International, Brea, CA, USA) at the Clinical Biochemical Laboratory of Xiangya Hospital (Changsha, China). The relative glucose consumption and lactate production rates were normalized by the protein concentration of samples.

Western blotting. Cells were harvested by trypsinization and pelleted by centrifugation. Cell pellets were lysed in Nonidet $\mathrm{P}-40$ cell lysis buffer (50 mM Tris- $\mathrm{Cl}, \mathrm{pH} 8.0,150 \mathrm{mM} \mathrm{NaCl}$, $0.5 \%$ Nonidet $\mathrm{P}-40$ and protease inhibitor mixture). Protein concentrations were determined using the Bradford assay (Bio-Rad, Hercules, CA, USA). Proteins were separated by SDS-PAGE and electrically transferred to a polyvinylidenedifluoride membrane (Millipore, Billerica, MA, USA). After blocking in 5\% non-fat dry milk in TBS, the membranes were hybridized to specific primary antibodies overnight at $4{ }^{\circ} \mathrm{C}$, washed three times with TBS Tween-20, and then incubated with secondary antibodies conjugated with horseradish peroxidase for $1 \mathrm{~h}$ at room temperature. The membranes were then washed three times in TBS Tween-20 at room temperature. The protein bands were visualized using ECL chemiluminescence reagents (Pierce Chemical Co., Rockford, IL, USA) according to the manufacturer's instructions.

Isolation of mitochondrial fractions. Following EGCG treatments, $\sim 5 \times 10^{6}$ cells from a $10-\mathrm{cm}$ plate were harvested by trypsinization and centrifuged at $800 \mathrm{rpm}$ for $5 \mathrm{~min}$ at $4^{\circ} \mathrm{C}$. The cell pellets were washed once with ice-cold PBS and then resuspended in three volumes of isolation buffer $(20 \mathrm{mM}$ HEPES, pH 7.4, $10 \mathrm{mM} \mathrm{KCl,} 1.5 \mathrm{mM} \mathrm{MgCl}_{2}, 1 \mathrm{mM}$ sodium EDTA, $1 \mathrm{mM}$ dithiothreitol, $10 \mathrm{mM}$ phenylmethylsulfonyl fluoride, $10 \mathrm{mM}$ leupeptin and $10 \mathrm{mM}$ aprotinin) in $250 \mathrm{mM}$ sucrose. After chilling on ice for $3 \mathrm{~min}$, the cells were disrupted by 60 strokes of a glass homogenizer. The homogenate was centrifuged once at $2,000 \mathrm{rpm}$ at $4^{\circ} \mathrm{C}$ for $10 \mathrm{~min}$ to remove unbroken cells and nuclei. The mitochondria-enriched fraction (supernatant) was then pelleted by centrifugation at 13,000 rpm for $30 \mathrm{~min}$. The pellets were lysed in RIPA buffer [10 mM Tris-Cl (pH 8.0), 1 mM EDTA, 0.5 mM EGTA, $1 \%$ Triton X-100, 0.1\% sodium deoxycholate, $0.1 \%$ SDS, $140 \mathrm{mM}$ $\mathrm{NaCl}]$, and analyzed by western blotting.

Soft agar colony formation assay. To assess the anchorageindependent growth, tongue carcinoma cells were suspended $\left(8 \times 10^{3}\right.$ cells $\left./ \mathrm{ml}\right)$ in $1 \mathrm{ml}$ of $0.3 \%$ agar with Eagle's basal medium containing $10 \%$ FBS, $1 \%$ antibiotics, and different concentrations of EGCG $(0,20,40$ and $80 \mu \mathrm{M} / \mathrm{l})$ overlaid in 6-well plates containing a $0.6 \%$ agar base. The cultures were maintained in a $37^{\circ} \mathrm{C}, 5 \% \mathrm{CO}_{2}$ incubator for $1-2$ weeks, and then colonies were counted under a microscope using the Image-Pro Plus software program (Media Cybernetics, Silver Spring, MD, USA).

Statistical analysis. Statistical analyses were performed using the SPSS software (version 13.0). The experiments were performed in triplicate. Quantitative data are presented as means \pm standard deviation. Significant differences between two groups were assessed by a two-tailed Student's t-test. $\mathrm{P}<0.05$ was considered to indicate a statistically significant difference.

\section{Results}

EGCG inhibits the anchorage-independent growth of human tongue carcinoma cells. Anchorage-independent growth is one of the malignant phenotypes of tumor cells and is considered to be one of the most accurate and stringent in vitro assays for detecting the malignant transformation of cells. Therefore, we first investigated the effect of EGCG on the anchorageindependent growth of human tongue carcinoma cells. Our results demonstrated that although EGCG had little effect on colony formation at $20 \mu \mathrm{M}$, the inhibition rate reached $>60 \%$ after Tca8113 cells exposure to EGCG at the concentration of $40 \mu \mathrm{M}$. More importantly, $80 \mu \mathrm{M}$ of EGCG almost blocked the colony growth in soft agar (Fig. 1A). We also found that EGCG 
A

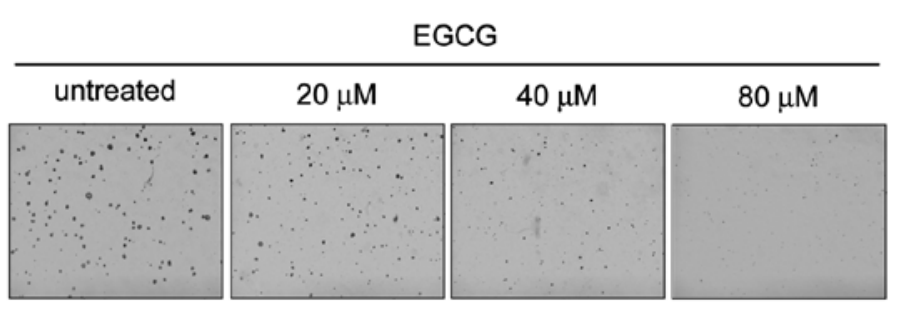

B

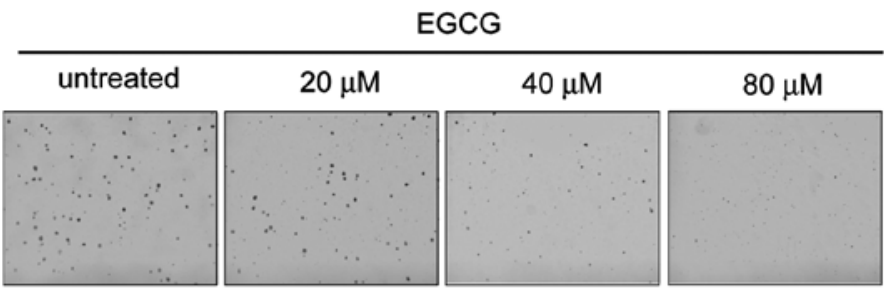

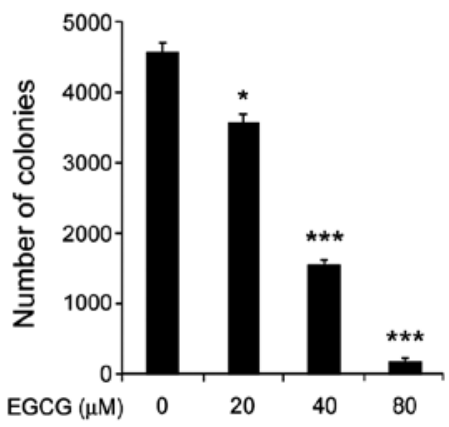

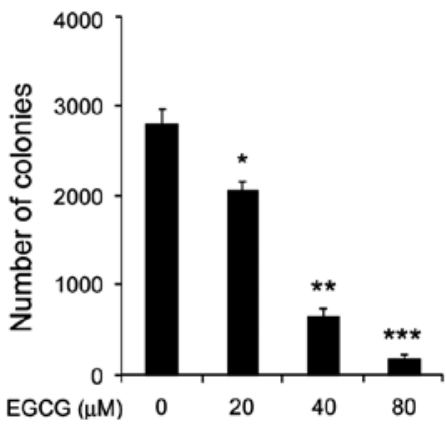

Figure 1. Inhibitory effects of EGCG on anchorage-independent growth of human tongue carcinoma cells. EGCG inhibits anchorage-independent growth of (A) Tca8113 and (B) TSCCa cells. A colony formation assay was performed as described in Materials and methods. Data show the colony formation ability of human tongue carcinoma cells treated with different concentrations of EGCG compared with the dimethyl sulfoxide-treated group. The average colony number was calculated from three independent experiments. Columns, mean of the number of colonies as determined from three independent experiments; bars, standard deviation; asterisk, significant $\left({ }^{*} \mathrm{p}<0.05,{ }^{* *} \mathrm{p}<0.01,{ }^{* * *} \mathrm{p}<0.001\right)$ suppression of colony formation by EGCG. EGCG, epigallocatechin gallate.
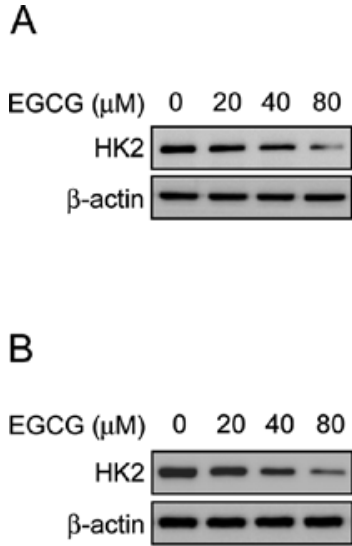
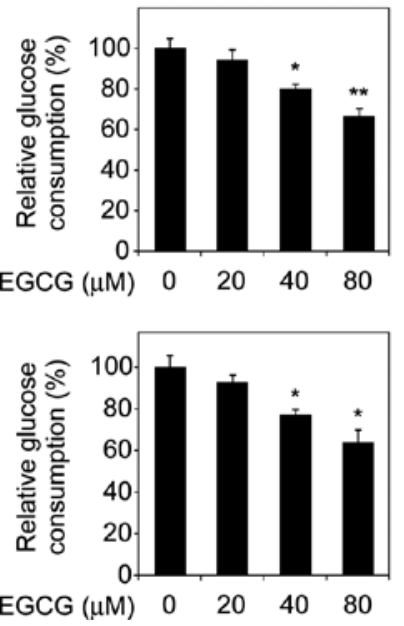
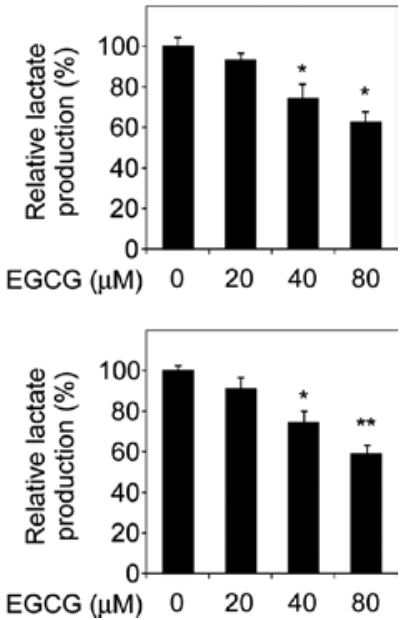

Figure 2. EGCG suppresses glycolysis in human tongue carcinoma cells. (A) EGCG inhibits glycolysis in Tca8113 cells. Western blotting was performed to detect HK2 in EGCG treatment of Tca8113 cells (left), the levels of glucose consumption (middle) and lactate production (right) in these cells. (B) EGCG inhibits glycolysis in TSCCa cells. Western blotting was performed to detect HK2 in EGCG treatment of TSCCa cells (left), the levels of glucose consumption (middle) and lactate production (right) in these cells. Data are shown as means \pm SD of three independent experiments. Significant $\left({ }^{*} \mathrm{p}<0.05,{ }^{* *} \mathrm{p}<0.01\right)$ decrease in glucose consumption or lactate production compared to EGCG untreated cells. EGCG, epigallocatechin gallate.

had a similar effect on the suppression of the anchorage-independent growth of TSCCa cells in soft agar (Fig. 1B). These data indicated that EGCG inhibits anchorage-independent growth of human tongue carcinoma cells in a dose-dependent manner.

EGCG downregulates HK2 expression and decreases human tongue carcinoma cell glycolysis. Elevated glycolysis is considered to be an emerging hallmark of human cancer.
HK2, a rate-limiting enzyme of glycolysis, is upregulated in various cancers (31). Thus, in the present study, we investigated whether EGCG-mediated antitumor activity is associated with tongue carcinoma cell glycometabolism. Immunoblot analysis indicated that the $\mathrm{HK} 2$ protein level decreased in response to EGCG treatment in Tca8113 cells (Fig. 2A, left) and a similar result was also observed in TSCCa cells (Fig. 2B, left). To assess the effect of EGCG on glycolysis, we examined the level of glucose consumption and lactate production in the two 
human tongue carcinoma cells. Consistent with the results of the immunoblot analysis, EGCG dose-dependently inhibited the consumption of glucose (Fig. 2A and B, middle) and production of lactate (Fig. 2A and B, right) in the Tca8113 and TSCCa cells. These results suggested that EGCG-mediated tumor suppression is partially dependent on glycolysis inhibition, and HK2 is involved in this process.

EGCG decreases HK2 expression by downregulating the EGFR-Akt signaling pathway. Findings of recent studies demonstrated that the Akt signaling pathway is a hub in the regulation of cancer metabolism $(36,37)$. As an Akt upstream receptor tyrosine kinase, the oncoprotein EGFR is always overexpressed in human tongue carcinoma (38). The results showed that EGCG strongly inhibited EGF-induced EGFR activation and EGCG also significantly inhibited the phosphorylation of EGFR downstream kinases Akt and ERK1/2. Furthermore, we found that the protein levels of HK2 were inhibited following EGCG treatment (Fig. 3A). To determine the connection between ERKs/Akt and HK2 suppression in the presence of EGCG, we used PD98059, a specific inhibitor of the ERKs pathway, to treat Tca8113 and TSCCa cells and examined whether HK2 expression would be affected by the inhibition of the ERKs signaling pathway in these cells. Results indicated that treatment with PD98059 markedly decreased the phosphorylation level of ERKs, however, there was no obvious effect on the expression of HK2 (Fig. 3B). By contrast, wortmannin, a specific inhibitor of the Akt pathway, not only substantially inhibited Akt activity, but also strongly suppressed HK2 expression in these cells (Fig. 3C). These results indicated that Akt, but not ERKs, was a key kinase of the EGCG-mediated downregulation of HK2.

Constitutively activated Akt1 rescues EGCG-mediated glycolysis inhibition. Based on the previous data, in order to confirm that the regulation of glycolysis by EGCG in human tongue carcinoma cells is dependent on Akt activity, we transfected constitutively activated Akt1 (Myr-Akt1) into Tca883 and TSCCa cells. The results indicated that $80 \mu \mathrm{M}$ EGCG significantly decreased the expression of HK2 in Tca8113 and TSCCa cells. However, Myr-Aktl transfection markedly upregulated HK2 expression in these EGCG-treated cells as expected (Fig. 4A and B, left). Moreover, Myr-Akt1 rescued over 70 and $60 \%$ of the deficient glucose uptake (Fig. 4A and B, middle) and lactate production (Fig. 4A and B, right) in EGCG-treated Tca8113 and TSCCa cells, respectively. These results suggested that EGCG regulates glycolysis in human tongue carcinoma cells, and these biochemical processes are partly mediated by Akt activation.

EGCG inhibits HK2 expression on mitochondrial outer membrane and induces apoptosis. The HK2 protein is crucial in cell proliferation as well as apoptosis resistance. In cancer cells, HK2 overexpression is associated with hyperactivated glycolysis and upregulated cell growth. More importantly, for HK2 to exert its anti-apoptotic function, HK2 translocates to the mitochondrial outer membrane and interacts with the voltage-dependent anion channel (VDAC) to block the release of cytochrome $c$, eventually inhibiting the apoptotic pathway (39-42). In order to assess the influence of EGCG
A

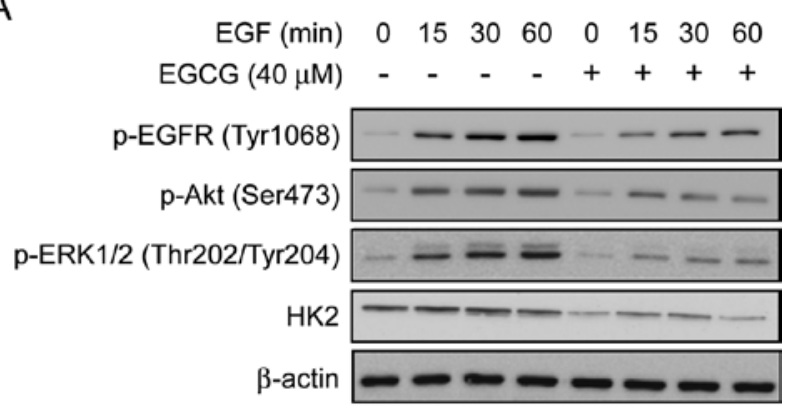

B

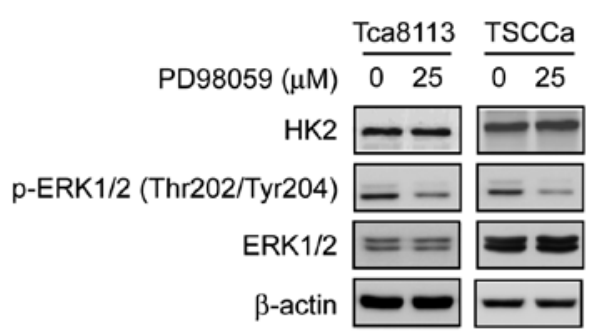

C

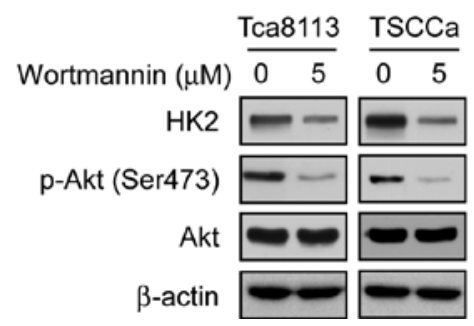

Figure 3. Akt signaling pathway is involved in HK2 regulation in human tongue carcinoma cells. (A) EGCG affects EGF-induced epidermal growth factor receptor signaling in Tca8113 cells. Tca8113 cells were starved in serum-free medium for $24 \mathrm{~h}$, following pre-treatment with or without $40 \mu \mathrm{M}$ EGCG for $6 \mathrm{~h}$. The cells were stimulated with EGF $(20 \mathrm{ng} / \mathrm{ml})$ at various time points, cell lysates were collected and protein levels were determined by western blot analysis. (B) ERK1/2 activity is dispensable for HK2 expression in human tongue carcinoma cells. Tca8113 and TSCCa cells were treated with/without $25 \mu \mathrm{M}$ PD98059 for $24 \mathrm{~h}$, and the protein levels of p-ERK1/2 and HK2 were tested by western blot analysis. (C) Akt phosphorylation is required for HK2 expression in human tongue carcinoma cells. Tca8113 and TSCCa cells were treated with or without $5 \mu \mathrm{M}$ wortmannin for $24 \mathrm{~h}$, and the protein levels of p-Akt and HK2 were detected by western blot analysis. EGCG, epigallocatechin gallate.

on the subcellular localization of HK2, mitochondrial fractions from Tca8113 and TSCCa cells, with or without $80 \mu \mathrm{M}$ EGCG treatment, were extracted and analyzed by immunoblotting with antibodies to detect HK2, $\alpha$-tubulin and VDAC. The results indicated that the translocation of HK2 in the mitochondrial outer membrane fraction was decreased in the Tca8113 and TSCCa cells following EGCG treatment (Fig. 5A). Furthermore, we evaluated the apoptotic signaling pathway by immunoblotting to examine the cleaved caspase-3 and cleaved poly(ADP-ribose) polymerase (PARP) expression after EGCG treatment. The results demonstrated that the levels of cleaved-caspase-3 and cleaved-PARP in Tca8113 and TSCCa cells were markedly increased in response to EGCG treatment (Fig. 5B). Based on these data, we suggested that the EGCG-mediated downregulation of HK2 inhibits anti-apoptotic effects of human tongue carcinoma cells. 

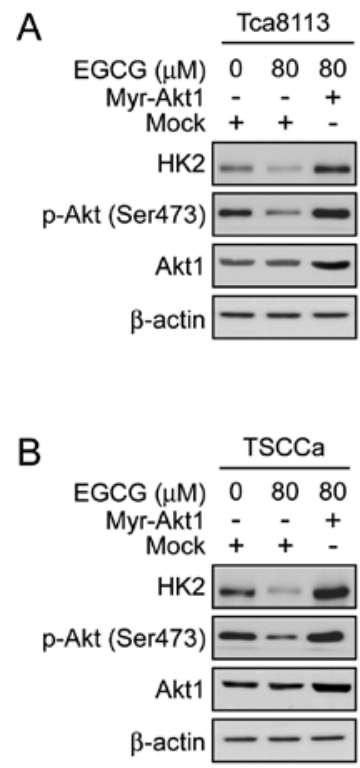
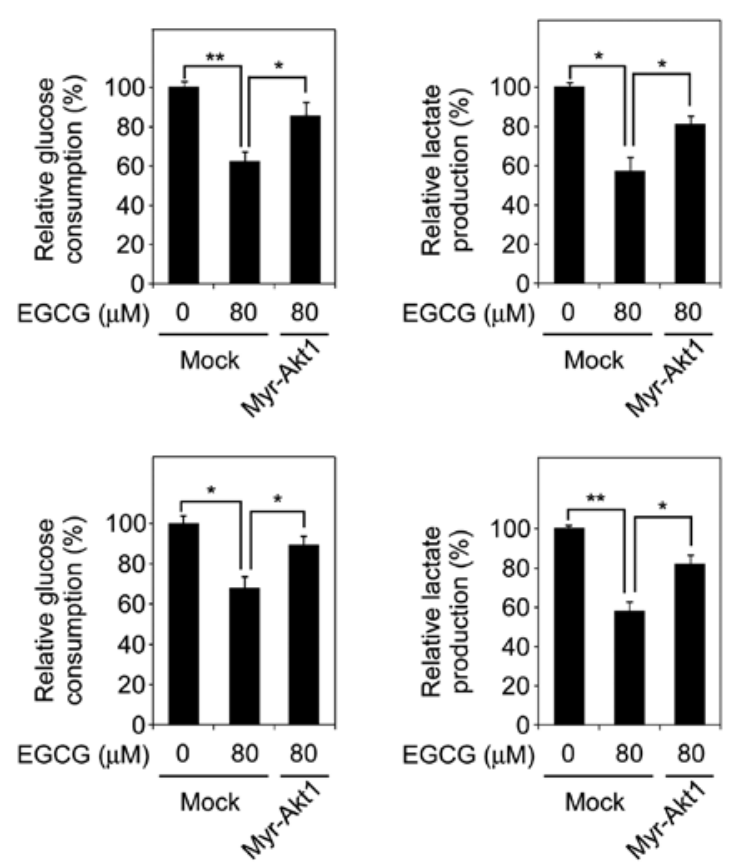

Figure 4. Overexpression of constitutively active Akt (Myr-Akt1) rescues glycolysis in EGCG-treated human tongue carcinoma cells. The Myr-Akt1 plasmid was transfected into (A) Tca8113 and (B) TSCCa cells as indicated. After transfection for $24 \mathrm{~h}$, the cells were treated with/without $80 \mu \mathrm{M}$ EGCG for another $24 \mathrm{~h}$. Western blot analysis was performed to detect HK2 expression (left). Glucose consumption (middle) and lactate production (right) were determined in these cells. Data are shown as means \pm SD of three independent experiments.Significant $\left({ }^{*} \mathrm{p}<0.05,{ }^{* *} \mathrm{p}<0.01\right)$ difference in glucose consumption or lactate production in these cells. EGCG, epigallocatechin gallate.

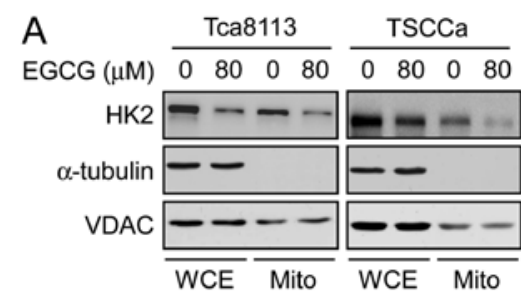

B

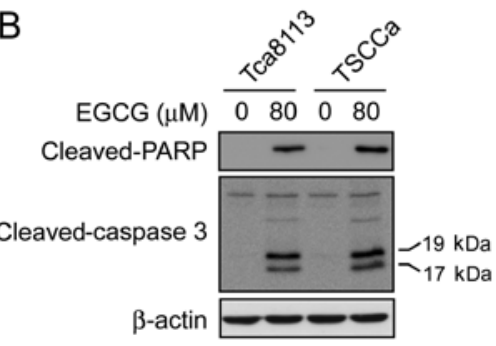

Figure 5. EGCG inhibits the translocation of HK2 to the mitochondrial outer membrane and induces apoptosis in human tongue carcinoma cells. (A) EGCG suppresses the translocation of HK2 to the mitochondrial outer membrane. Tca8113 and TSCCa human tongue carcinoma cells were treated with/without $80 \mu \mathrm{M}$ EGCG for $24 \mathrm{~h}$, and the effect of EGCG on the translocation of HK2 to the mitochondrial outer membrane was determined by immunoblot analysis. (B) EGCG treatment promotes human tongue carcinoma cell apoptosis. Tca8113 and TSCCa cells were treated with/without $80 \mu \mathrm{M}$ EGCG for $24 \mathrm{~h}$, cleaved PARP and caspase- 3 were analyzed by immunoblot analysis. EGCG, epigallocatechin gallate.

\section{Discussion}

Oral cavity cancer is ranked as the sixth most widespread cancer type worldwide. Carcinoma of the oral tongue, the most common cancer in the oral cavity, exhibits invasion and/or metastasis at a very early stage. Surgery, radiotherapy, chemotherapy and combined modalities are the main treatment options for the tongue carcinoma (21-24). However, no molecular-targeted therapeutic drugs against tongue carcinoma are currently licensed, and the prevention strategies to decrease the incidence remain elusive. The limited therapeutic options provide a strong stimulus for the development of novel therapeutics. Preclinical studies have consolidated activity of EGCG in cancer prevention and, it was shown that inhibition of EGCG-induced proliferation and induction of apoptosis played an important role in its antitumor activity (5). However, how the underlying mechanisms of EGCG exert a tumor-suppressor effect through the metabolic pathways remains to be determined. We have demonstrated that EGCG had a profound antitumor activity in human tongue carcinoma cells in vitro. EGCG treatment decreased the expression of HK2 and inhibited its translocation into the mitochondrial outer membrane, while EGCG exerted its function in an Akt activity suppression-dependent manner.

In the present study, we first examined the antitumor activity of EGCG in two tongue carcinoma cells. The results indicated that EGCG markedly inhibited anchorage-independent growth of Tca8113 and TSCCA cells in a dose-dependent manner (Fig. 1A and B). These data are consistent with those of a previous study which reported that tea polyphenols have an inhibitory effect on the growth of oral squamous carcinoma cells (43). For cancer cells to sustain their rapid proliferation and gain a survival advantage, glycolysis has been demonstrated as a hot spot for metabolic reprogramming in tumor. This physiological process generates ATP for cell energy supply and provides sufficient biosynthetic intermediates for 
anabolic pathways (28). Although metabolic control over the glycolytic rate can be applied at many steps in the glycolytic pathway, most studies in cancer support the hypothesis that control over glycolytic flux primarily resides at the transport and phosphorylation steps (31). Findings of previous studies indicated that EGCG is associated with glucose uptake inhibition by regulating glucose transporter GLUTs, in different signaling pathways (44-47). Those findings suggested that EGCG may be involved in glycolysis regulation. Our results clearly showed that EGCG dose-dependently inhibited glucose consumption and lactate production in Tca8113 and TSCCa cells. Moreover, we found that EGCG directly decreased the expression of HK2 in these cells. These results suggest that EGCG regulates glycolysis in human tongue carcinoma cells.

HK2, the first rate-limiting enzyme in glycolysis, is considered to be required for tumor initiation and maintenance. Although the underlying mechanisms of the deregulation of HK2 in cancer cells have not yet been completely elucidated, the PI3-K/Akt-related signaling pathway, as well as the transcription factors, hypoxia inducible factors- $1 \alpha(\mathrm{HIF}-1 \alpha)$ and c-myc, have been demonstrated to be involved in the regulation of HK2 and HK2-mediated glycolysis (31). More importantly, overexpression of the receptor tyrosine kinase EGFR, accompanied by Akt hyperactivation is always observed in human tongue carcinoma and associated with tumor cell malignant properties $(38,48)$. Thus, we hypothesized that the EGFR-Akt signaling pathway may regulate glycolysis in human tongue carcinoma. Our results show that EGCG substantially inhibited EGF-induced EGFR and its downstream kinase Akt and ERK phosphorylation as well as HK2 expression as expected. Subsequent analysis verified that the downregulation of HK2 in Tca8113 and TSCCa cells is mediated through the Akt signaling pathway, but is not dependent on ERK activity. In order to confirm our observations, a transient transfection of constitutively activated Akt1 (Myr-Akt1) was conducted in EGCG-treated human tongue carcinoma cells. Our results have demonstrated that Myr-Akt1 transfection promoted the expression of HK2 and rescued the glucose consumption and lactate production in Tca8113 and TSCCa cells, although in the presence of EGCG. These findings provide direct evidence that downregulation of Akt signaling pathway is involved in the suppression of EGCG-induced human tongue carcinoma glycolysis.

Findings of laboratory and clinical investigations have demonstrated that the high expression of HK2 is associated with resistance to cancer cell apoptosis $(39,40)$. In this case, HK2 translocates to the mitochondrial outer membrane and interacts with the VDAC to block the release of cytochrome $c$, eventually inhibiting apoptosis (39-42). Our results indicate that EGCG obviously inhibited HK2 expression on the mitochondrial outer membrane and markedly promoted tongue carcinoma cell apoptosis. These results reveal a previously unknown mechanism of EGCG-induced tumor cell apoptosis, which may be partially dependent on HK2 subcellular localization. In summary, the present study suggests that glycolysis is involved in EGCG-mediated antitumor activity, and identified HK2 as a new potential target of EGCG. These results suggest that HK2 is a good molecular target for the prevention and treatment of human tongue carcinoma.

\section{Acknowledgements}

This study was supported by the National Natural Science Foundation of China (grant no. 81371690), the International Cooperation Program Funds of the China Hunan Provincial Science and the Technology Department (project nos. 2012WK4005 and 2013FJ6009).

\section{References}

1. Dreosti IE, Wargovich MJ and Yang CS: Inhibition of carcinogenesis by tea: the evidence from experimental studies. Crit Rev Food Sci Nutr 37: 761-770, 1997.

2. Katiyar S and Mukhtar H: Tea in chemoprevention of cancer. Int J Oncol 8: 221-238, 1996.

3. Yang CS, Maliakal P and Meng X: Inhibition of carcinogenesis by tea. Annu Rev Pharmacol Toxicol 42: 25-54, 2002.

4. Katiyar SK and Elmets CA: Green tea polyphenolic antioxidants and skin photoprotection (Review). Int J Oncol 18: 1307-1313, 2001.

5. Yang CS: Inhibition of carcinogenesis by tea. Nature 389: 134-135, 1997.

6. Yang CS and Wang H: Mechanistic issues concerning cancer prevention by tea catechins. Mol Nutr Food Res 55: 819-831, 2011.

7. Ma YC, Li C, Gao F, et al: Epigallocatechin gallate inhibits the growth of human lung cancer by directly targeting the EGFR signaling pathway. Oncol Rep 31: 1343-1349, 2014.

8. Maruyama T, Murata S, Nakayama K, et al: (-)-Epigallocatechin3 -gallate suppresses liver metastasis of human colorectal cancer. Oncol Rep 31: 625-633, 2014.

9. Bode AM and Dong Z: Epigallocatechin 3-gallate and green tea catechins: united they work, divided they fail. Cancer Prev Res 2: 514-517, 2009.

10. Shirakami Y, Shimizu M and Moriwaki H: Cancer chemoprevention with green tea catechins: from bench to bed. Curr Drug Targets 13: 1842-1857, 2012.

11. Singh BN, Shankar S and Srivastava RK: Green tea catechin, epigallocatechin-3-gallate (EGCG): mechanisms, perspectives and clinical applications. Biochem Pharmacol 82: 1807-1821, 2011.

12. Shimizu M, Shirakami Y and Moriwaki H: Targeting receptor tyrosine kinases for chemoprevention by green tea catechin, EGCG. Int J Mol Sci 9: 1034-1049, 2008.

13. Khoi PN, Park JS, Kim JH, et al: (-)-Epigallocatechin-3-gallate blocks nicotine-induced matrix metalloproteinase- 9 expression and invasiveness via suppression of NF- $\mathrm{KB}$ and AP-1 in endothelial cells. Int J Oncol 43: 868-876, 2013.

14. Dong Z, Ma W, Huang C and Yang CS: Inhibition of tumor promoter-induced activator protein 1 activation and cell transformation by tea polyphenols, (-)-epigallocatechin gallate, and theaflavins. Cancer Res 57: 4414-4419, 1997.

15. Rouzer CA and Marnett LJ: Green tea gets molecular. Cancer Prev Res 4: 1343-1345, 2011.

16. Bode AM and Dong Z: Targeting signal transduction pathways by chemopreventive agents. Mutat Res 555: 33-51, 2004.

17. Jankun J, Selman SH, Swiercz R and Skrzypczak-Jankun E: Why drinking green tea could prevent cancer. Nature 387: 561, 1997.

18. He Z, Tang F, Ermakova S, et al: Fyn is a novel target of (-)-epigallocatechin gallate in the inhibition of JB6 Cl41 cell transformation. Mol Carcinog 47: 172-183, 2008.

19. Sakamoto Y, Terashita N, Muraguchi T, Fukusato T and Kubota S: Effects of epigallocatechin-3-gallate (EGCG) on A549 lung cancer tumor growth and angiogenesis. Biosci Biotechnol Biochem 77: 1799-1803, 2013.

20. Katiyar S, Elmets CA and Katiyar SK: Green tea and skin cancer: photoimmunology, angiogenesis and DNA repair. J Nutr Biochem 18: 287-296, 2007.

21. Scully $C$ and Bagan J: Oral squamous cell carcinoma overview. Oral Oncol 45: 301-308, 2009.

22. Shah JP and Gil Z: Current concepts in management of oral cancer - surgery. Oral Oncol 45: 394-401, 2009.

23. Calabrese L, Tagliabue M, Maffini F, Massaro MA and Santoro L: From wide excision to a compartmental approach in tongue tumors: what is going on? Curr Opin Otolaryngol Head Neck Surg 21: 112-117, 2013. 
24. Yuen AP, Lam KY, Chan AC, et al: Clinicopathological analysis of elective neck dissection for N0 neck of early oral tongue carcinoma. Am J Surg 177: 90-92, 1999.

25. Rana M, Iqbal A, Warraich R, Ruecker M, Eckardt AM and Gellrich NC: Modern surgical management of tongue carcinoma - a clinical retrospective research over a 12 years period. Head Neck Oncol 3: 43, 2011.

26. Petersen PE: Global policy for improvement of oral health in the 21st century - implications to oral health research of World Health Assembly 2007, World Health Organization. Community Dent Oral Epidemiol 37: 1-8, 2009.

27. Preis M, Hadar T, Soudry E, et al: Early tongue carcinoma: analysis of failure. Head Neck 34: 418-421, 2012.

28. Koppenol WH, Bounds PL and Dang CV: Otto Warburg's contributions to current concepts of cancer metabolism. Nat Rev Cancer 11: 325-337, 2011.

29. Hanahan D and Weinberg RA: Hallmarks of cancer: the next generation. Cell 144: 646-674, 2011.

30. Tennant DA, Durán RV and Gottlieb E: Targeting metabolic transformation for cancer therapy. Nat Rev Cancer 10: 267-277, 2010.

31. Denko NC: Hypoxia, HIF1 and glucose metabolism in the solid tumour. Nat Rev Cancer 8: 705-713, 2008.

32. Wallace DC: Mitochondria and cancer: Warburg addressed. Cold Spring Harb Symp Quant Biol 70: 363-374, 2005.

33. Ros S and Schulze A: Glycolysis back in the limelight: systemic targeting of HK2 blocks tumor growth. Cancer Discov 3: 1105-1107, 2013.

34. Patra KC, Wang Q, Bhaskar PT, et al: Hexokinase 2 is required for tumor initiation and maintenance and its systemic deletion is therapeutic in mouse models of cancer. Cancer Cell 24: 213-228, 2013.

35. Wolf A, Agnihotri S, Micallef J, et al: Hexokinase 2 is a key mediator of aerobic glycolysis and promotes tumor growth in human glioblastoma multiforme. J Exp Med 208: 313-326, 2011.

36. Yecies JL and Manning BD: Transcriptional control of cellular metabolism by mTOR signaling. Cancer Res 71: 2815-2820, 2011

37. Fruman DA and Rommel C: PI3K and cancer: lessons, challenges and opportunities. Nat Rev Drug Discov 13: 140-156, 2014.

38. Ribeiro FA, Noguti J, Oshima CT and Ribeiro DA: Effective targeting of the epidermal growth factor receptor (EGFR) for treating oral cancer: a promising approach. Anticancer Res 34: $1547-1552,2014$
39. Cheung EC, Ludwig RL and Vousden KH: Mitochondrial localization of TIGAR under hypoxia stimulates HK2 and lowers ROS and cell death. Proc Natl Acad Sci USA 109: 20491-20496, 2012.

40. Pastorino JG, Shulga N and Hoek JB: Mitochondrial binding of hexokinase II inhibits Bax-induced cytochrome $c$ release and apoptosis. J Biol Chem 277: 7610-7618, 2002.

41. Majewski N, Nogueira V, Bhaskar P, et al: Hexokinasemitochondria interaction mediated by Akt is required to inhibit apoptosis in the presence or absence of Bax and Bak. Mol Cell 16: 819-830, 2004

42. Krasnov GS, Dmitriev AA, Lakunina VA, Kirpiy AA and Kudryavtseva AV: Targeting VDAC-bound hexokinase II: a promising approach for concomitant anti-cancer therapy. Expert Opin Ther Targets 17: 1221-1233, 2013.

43. Elattar TM and Virji AS: Effect of tea polyphenols on growth of oral squamous carcinoma cells in vitro. Anticancer Res 20: 3459-3465, 2000.

44. Moreira L, Araújo I, Costa T, et al: Quercetin and epigallocatechin gallate inhibit glucose uptake and metabolism by breast cancer cells by an estrogen receptor-independent mechanism. Exp Cell Res 319: 1784-1795, 2013.

45. Ueda M, Nishiumi S, Nagayasu H, Fukuda I, Yoshida K and Ashida H: Epigallocatechin gallate promotes GLUT4 translocation in skeletal muscle. Biochem Biophys Res Commun 377: 286-290, 2008.

46. Ku HC, Tsuei YW, Kao CC, et al: Green tea (-)-epigallocatechin gallate suppresses IGF-I and IGF-II stimulation of 3T3-L1 adipocyte glucose uptake via the glucose transporter 4 , but not glucose transporter 1 pathway. Gen Comp Endocrinol 199: 46-55, 2014.

47. Naftalin RJ, Afzal I, Cunningham P, et al: Interactions of androgens, green tea catechins and the antiandrogen flutamide with the external glucose-binding site of the human erythrocyte glucose transporter GLUT1. Br J Pharmacol 140: 487-499, 2003.

48. Ongkeko WM, Altuna X, Weisman RA and Wang-Rodriguez J: Expression of protein tyrosine kinases in head and neck squamous cell carcinomas. Am J Clin Pathol 124: 71-76, 2005. 